\title{
Bee Mix Antigen IgE Antibody Measurement
}

National Cancer Institute

\section{Source}

National Cancer Institute. Bee Mix Antigen IgE Antibody Measurement. NCI Thesaurus.

Code C130116.

A measurement of the bee mix antigen IgE antibody in a biological specimen. 